NASA Contractor Report 204138

ICOMP-97-07; CMOTT-97-02

AIAA-97-3115

\title{
Automated Simplification of Full Chemical Mechanisms
}

\author{
A.T. Norris \\ Institute for Computational Mechanics in Propulsion \\ and the Center for Modeling of Turbulence and Transition \\ Cleveland, Ohio
}

August 1997

Prepared for

Lewis Research Center

Under Cooperative Agreement NCC3-534

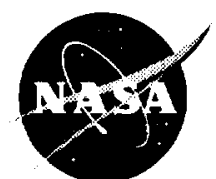

National Aeronautics and

Space Administration

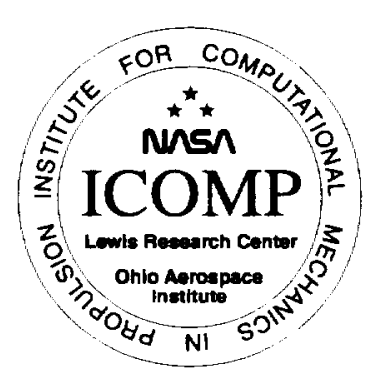




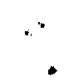

.

$\checkmark$ 


\title{
AUTOMATED SIMPLIFICATION OF FULL CHEMICAL MECHANISMS
}

\author{
A. T. Norris * \\ Institute for Computational Mechanics in Propulsion \\ and the Center for Modeling of Turbulence and Transition \\ NASA Lewis Research Center, Cleveland Ohio.
}

\begin{abstract}
A code has been developed to automatically simplify full chemical mechanisms. The method employed is based on the Intrinsic Low Dimensional Manifold (ILDM) method of Maas and Pope ${ }^{1,2}$.

The ILDM method is a dynamical systems approach to the simplification of large chemical kinetic mechanisms. By identifying low-dimensional attracting manifolds, the method allows complex full mechanisms to be parameterized by just a few variables: In effect, generating reduced chemical mechanisms by an automatic procedure. These resulting mechanisms however, still retain all the species used in the full mechanism.

Full and skeletal mechanisms for various fuels are simplified to a two dimensional manifold, and the resulting mechanisms are found to compare well with the full mechanisms, and show significant improvement over global one step mechanisms, such as those by Westbrook and Dryer ${ }^{3}$. In addition, by using an ILDM reaction mechanism in a CFD code, a considerable improvement in turn-around time can be achieved.
\end{abstract}

\section{Introduction}

Detailed chemical mechanisms for hydrocarbon fuels typically contain in order of 50 species and 200

* Senior Research Associate, Member AIA A

Copyright (c) 1997 by the American Institute of Aeronautics and Astronautics, Inc. No copyright is asserted in the United States under Title 17, U.S. Code. The U.S. Government has a royalty-free licence to exercise all rights under the copyright claimed herein for Governmental Purposes. All other rights are reserved by the copyright owner. rate equations. Applying these mechanisms to a $3 \mathrm{D}$ turbulent combustion problem is beyond the capabilities of todays computers, and beyond those in the foreseeable future. Thus the practice of simplifying the full mechanisms is used, where the number of species and equations is reduced by various assumptions about the reaction. e.g. Partial equilibrium of species. In the crudest sense, this reduction is achieved by throwing out, or combining species and rate equations.

However to perform these simplifications, considerable human input is required, both in knowledge of the detailed mechanism and testing the resulting reduced mechanism. In addition, the accuracy of the reduced mechanism drops with every simplification, requiring the user to compromise between how much accuracy they need against how much they can afford.

This need for considerable human effort to obtain reduced mechanisms was the motivation for the development of the ILDM method ${ }^{1,2}$ of simplifying chemical kinetics. The objectives were to provide a completely automated method of simplifying chemical kinetics, with the only input being the full mechanism, and the degree of simplification.

In this paper, the development of an ILDM code will be described. This code will be used to simplify several hydrocarbon mechanisms, and the performance of these simplifications will be tested in a Perfectly Stirred Reactor (PSR) code. The ILDM results will be compared against the full mechanism, a 12 species, 10 step reduced mechanism and a one step mechanism. In addition, the performance of the reduced mechanism in a CFD code will be investigated. 


\section{ILDM Method}

The composition of a chemical system can be thought of as a point in a multidimensional space: each dimension representing a species concentration. Thus the rate equations can be thought of as representing how that point moves in the scalar space. The ILDM approach is based on analysing and simplifying these equations of motion of the scalar point.

How the ILDM method does this is to identify attracting manifolds in the scalar space. Because of the wide range of time scales in a chemical system, the composition changes very rapidly in some directions and moves slowly in others. Thus the composition moves rapidly toward the attracting manifold, and then moves along that surface to the equilibrium point. To simplify the mechanism, one simply assumes the composition always lies on these manifolds. Thus all one need do is specify the dimension of the manifold. For example, a zero-dimensional manifold is simply the equilibrium point while a one-dimensional manifold is a line, on which lies the equilibrium point.

Because a one-dimensional manifold is just a line in the scalar space, it can be parameterized by just one variable. This variable can be a scalar, a combination of scalars or even a thermodynamic property, and is referred to as the reference variable. The only requirement is that the reference variable does not give a multi-valued function.

One interesting benefit of this method of simplification is that the value of all the species in the full mechanism on the manifold are available, as a function of the reference variable. Thus the minor, and trace species are available without any increase in the complexity of the reaction mechanism. This is contrasted with conventional reduced mechanisms that require additional species and equations to be added if more detailed composition information is required. In addition, state properties can also be stored on the manifold, and so properties like density are calculated with all the minor species being taken into account, thus providing a more accurate value.

In the code developed at Lewis, the ILDM simplified mechanism is obtained by a trajectorygenerated technique, described by Pope and Maas 4. In this method, the full mechanism is parameterized by two scalars, a mixture fraction based on atom concentrations and a progress variable, based on either species mass fractions, temperature or Gibbs function. The resulting species concentrations, rates and properties are stored in look-up tables. Also provided is a set of interpolation routines that can be called from CFD codes. These provide the interface between the ILDM tables and the combustion code.

\section{Reaction Mechanisms}

In this paper, three hydrocarbon fuels are studied: methane, propane and Jet $\mathrm{A}$. The mechanisms used are the methane mechanism of Miller and Bowman ${ }^{5}$, the propane mechanism of Jachimowski ${ }^{6}$ and the Jet A mechanism of Kollrack ${ }^{7}$.

In addition, two reduced mechanisms are used for comparison. These are the one step methane mechanism of Westbrook and Dryer ${ }^{3}$ and the 12 step methane mechanism of Kundu ${ }^{8}$.

\section{Test Case}

The test case employed for the reaction kinetics is the Perfectly Stirred Reactor (PSR). In this example, a mixture of fuel and air flows into a reactor vessel. This mixture mixes instantly with the composition in the container, reacts and the resulting products exit the container. Residence time is defined as the density of the exit composition multiplied by the volume of the reactor, divided by the mass flow rate of the system.

Initial conditions are chosen to be those similar to gas turbine operation, with fuel and air inlet temperatures at $350 \mathrm{~K}$ and $750 \mathrm{~K}$ respectively, and a pressure of 10 atmospheres.

\section{Results}

Results will consist of plots of residence time vs. property for the several different reaction schemes and for different inlet mixture fractions. Due to the quantity of results available, only a selection will be presented. In all these cases, the progress variable (Yp) chosen is the sum of the mass fractions of $\mathrm{CO} 2$ and $\mathrm{H} 2 \mathrm{O}$.

The first area addressed is the table resolution. i.e. How many grid points are required to accurately represent the reaction scheme. A stoichiometric mixture of methane and air was chosen as the representive composition for this test. 
In Fig. 1, the results for refinement of the table in the Yp direction are shown against the full mechanism. It can be seen that having 32 points provides very good agreement with the full mechanism for all species.

In Fig. 2, the effect of table resolution in the mixture fraction (xi) direction is investigated. It can be seen that a grid spacing of 0.0025 provides a very good agreement with the full mechanism. This corresponds to 24 grid nodes between the flamibility limits. Considering these results, a grid resolution of $32 \times 32$ is used for the remaining cases.

In Fig. 3 and 4, the performance of the ILDM table is shown compared to the full mechanism ${ }^{5}$, a 12 species, 10 step mechanism ${ }^{8}$ and a one step mechanism ${ }^{3}$. It can be seen that the ILDM scheme compares very well with the full mechanism and the 10 step scheme in all plots. Especially noteworthy is the very good agreement with the density, as this variable is the one that affects the flow calculations. The poor agreement by the one-step mechanism can be attributed to the lack of minor species in that mechanism. It should be noted however that the results for the mass fraction of fuel show a very poor agreement. This lack of agreement is present in all the cases tested, and will be discussed in the next section.

The same fuel was then used, but for lean and rich mixtures. The results of these are shown in Fig. 5. Both give good agreement with the full mechanism.

In Fig. 6, the results for a stoichiometric mixture of propane and air are shown. Again the results are in good agreement with the full mechanism. The only difference observed is the kink in the $Y_{\mathrm{H}_{2} \mathrm{O}}$ result for small residence times. However the very good agreement of the density result shown that this would not have a noticeable effect on the overall performance of the table.

However the results for JetA, shown in Fig. 7 show a divergence from the full mechanism results at short residence times. This can be probably be attributed to the full mechanism used in this test. The mechanism used ${ }^{7}$ is the smallest of the three mechanisms, despite being the most complex fuel. The problem may lie in the omission of some important intermediate species. In addition, this mechanism is the oldest of all three. Comparing the elementary rate constants with those of methane and propane reveal many differences. It is quite possible that the rate constants of some intermediate species may be in error, thus affecting the high-load results.

The next part of the mechanism investigated was the speed of the look-up table compared to using an equivalent reduced mechanism. To perform this test, the 12 species methane mechanism used in a combustion code was analyzed. For a 500,000 node problem, the CFD run-time was about 14.0 seconds per iteration, using 84 nodes on an SP2 machine. Of that time, 7.0 seconds was spent in performing the reaction and calculating the composition properties.

In this code, the equivalent look-up table requirements would require $0.17 \mathrm{sec}$. In addition, because the transport equations for only mixture fraction and progress variable would need to be calculated, rather than 12 species, it is estimated that the time saved using the lookup tables would be about 10.7 seconds per iteration.

The space required for these look-up tables is estimated as $4.3 \mathrm{KBytes}$ per property stored. This gives a total table size of about $60 \mathrm{KBy}$ tes.

\section{Discussion}

A feature of the results that needs to be discussed is the poor prediction of fuel concentrations in all three cases.

For the fuel prediction, the lack of agreement between the full mechanism results and the reduced mechanisms is a function of the simplification procedure. Put simply, the break-up of the fuel molecule is one of the processes that the ILDM scheme deems to occur very rapidly, and so the PSR results show essentially zero concentration until near extinction. However the performance of the model, specifically in the prediction of density, shows that the error in predicting the fuel concentrations does not compromise the overall accuracy of the model.

Another area to be addressed is the implementation of the ILDM mechanism in a CFD code.

The current methodology used is to store the mechanism in a simple look-up table as a function of the mixture fraction and the progress variable. The look-up table consists of a uniform grid, bounded in the mixture fraction direction by the lean and rich flamability limits. In the progress variable direction it is bounded by the pure mixing and fully burnt limits. When used in a CFD code, it is found that the best way to implement the scheme is by storing 
the reaction increments for a set of different time steps. In addition the properties such as density, laminar viscosity and thermal diffusivity are also stored in the table. Thus the interpolation routine is given an initial composition and time step, and it returns the updated composition, along with the new property values.

It should also be noted that the current implementation of the ILDM scheme contains some limitations to its applicability. The assumptions made for the current model are constant pressure, unity Lewis number and simple inlet conditions. i.e. All fuel inlet temperatures and compositions must be the same, and all air inlet temperatures must have their temperatures and compositions identical. However these are just restrictions of the current implementation, not of the ILDM scheme in general. By including pressure as a tabulation variable, the uniform pressure restriction can be lifted. By including other variables such as enthalpy and composition the other cases can also be solved for by this method.

\section{Conclusion}

In this paper it has been shown that a 1-step ILDM scheme gives good agreement with full mechanism results in the PSR test for a variety of hydrocarbon fuels. The accuracy of the method has also been shown to be similar similar to that of a 12 species, 10 step mechanism. In addition due to the tabular nature of the storage, the ILDM scheme can provide significant savings in CPU time when coupled with a CFD code. While the current implementation does restrict the class of problem that can be treated by this method, it should be noted that relatively simple changes to the storage methods can overcome these.

\section{References}

1. U. A. Maas and S. B. Pope. Simplifying chemical kinetics: Intrinsic low-dimensional manifolds in composition space. Combustion and Flame, $\mathbf{8 8}(3 / 4): 239-264,1992$.

2. U. Maas and S. B. Pope. Laminar flame calculations using simplified chemical kinetics based on intrinsic low-dimensional manifolds. In TwentyFifth Symposium (International) on Combustion, page In Press, 1994.
3. C. K. Westbrook and F. L. Dryer. Chemical kinetic modeling of hydrocarbon combustion. Prog. Energy Combust. Sci., 10:1-57, 1984.

4. S. B. Pope and U. Maas. Simplifying chemical kinetics: Trajectory-generated low-dimensional manifolds. Technical Report FDA-93-11, Sibley School of Mechanical and Aerospace Engineering: Fluid dynamics and aerodynamics program, 1993.

5. J. A. Miller and C. T. Bowman. Mechanism and modeling of nitrogen chemistry in combustion. Prog. Energy Combust. Sci, 15 :287-338, 1989.

6. C. J. Jachimowski. Chemical kinetic mechanism for the combustion of propane. Combustion and Flame, 55:213-224, 1984.

7. R. Kollrack. Model calculations of the combustion product distributions in the primary zone of a gas turbine combustor. In ASME Winter Annual Meeting, New York NY, pages ASME 76-WA/GT-7, 1976.

8. K. Kundu. Methane 12 species reduced mechanism. Private Comunication, 1996.

\section{Acknowledgements}

The author would like to acknowledge the assistance of Dr. K. Kundu of NASA Lewis Research Center for use of his unpublished (so far!) 12 species reduced methane-air mechanism. Also thanks are extended to Dr. A. Brankovic of Pratt and Whitney for finding the JetA mechanism, and Dr. J. Deur, formerly of NASA Lewis, for obtaining the propane mechanism. 

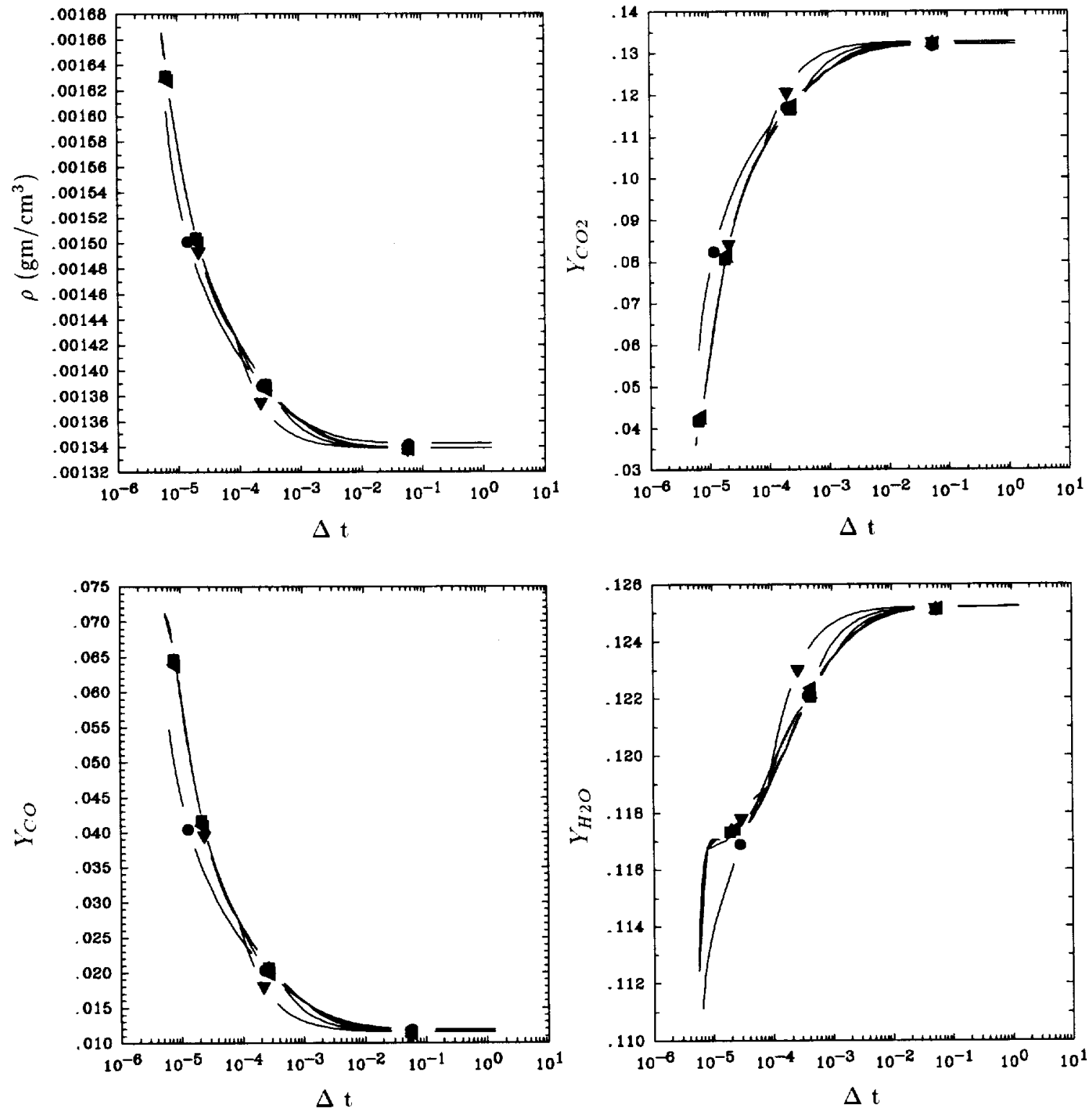

Figure 1: Test of table resolution in $Y_{P}$ direction for stoichiometric methane-air mixture. PSR results shown as composition quantities plotted against residence time. $\bigcirc$ - Full mechanism; $\square-64$ nodes; $\triangle$ 32 nodes; $\triangleleft-16$ nodes; $\nabla-8$ nodes. 

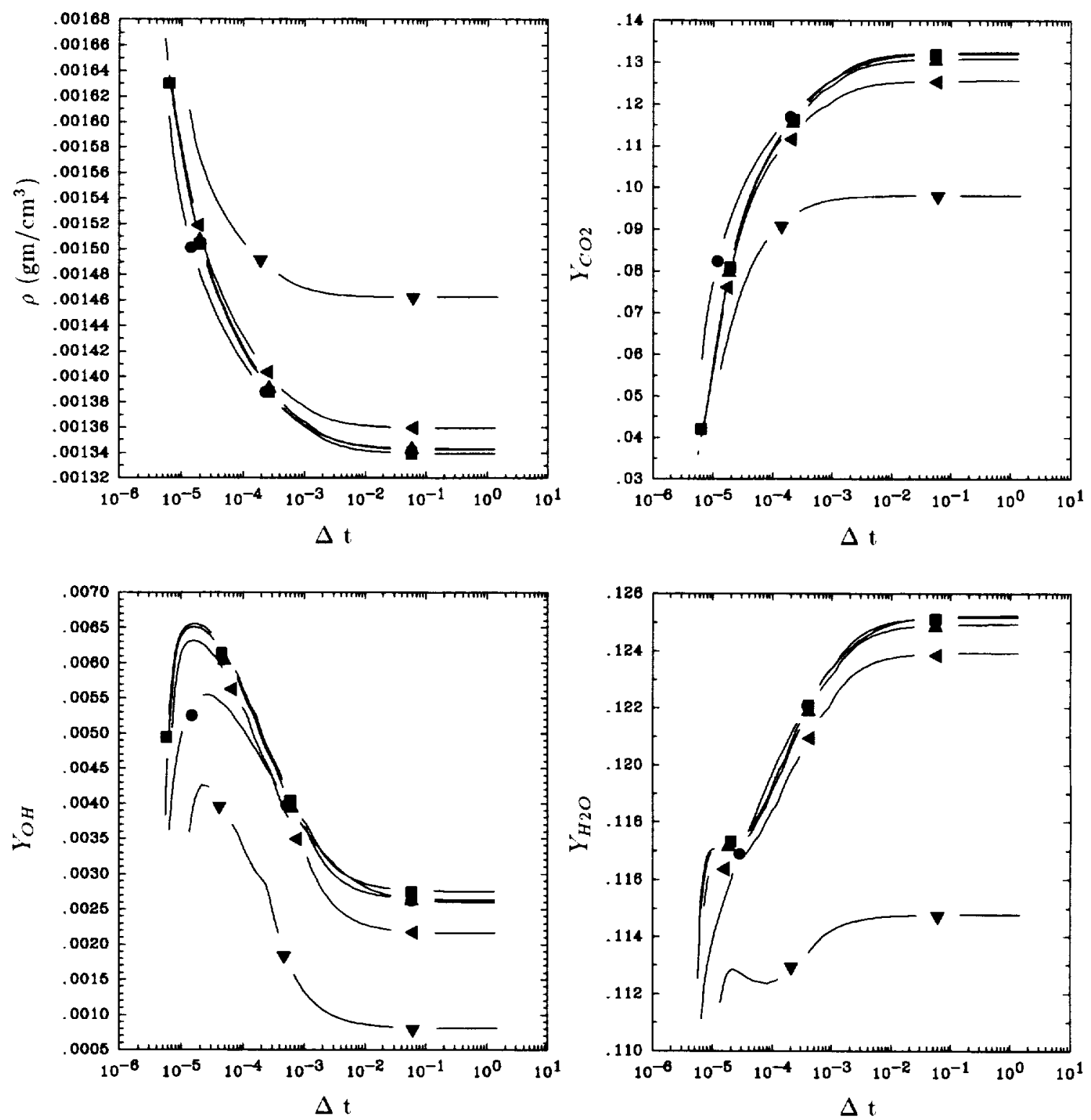

Figure 2: Test of table resolution in $x i$ direction for stoichiometric methane-air mixture. PSR results shown as composition quantities plotted against residence time. $\bigcirc$ - Full mechanism; $\square-0.0025$ spacing; $\triangle$ 0.005 spacing; $\triangleleft-0.01$ spacing; $\nabla-0.02$ spacing. 


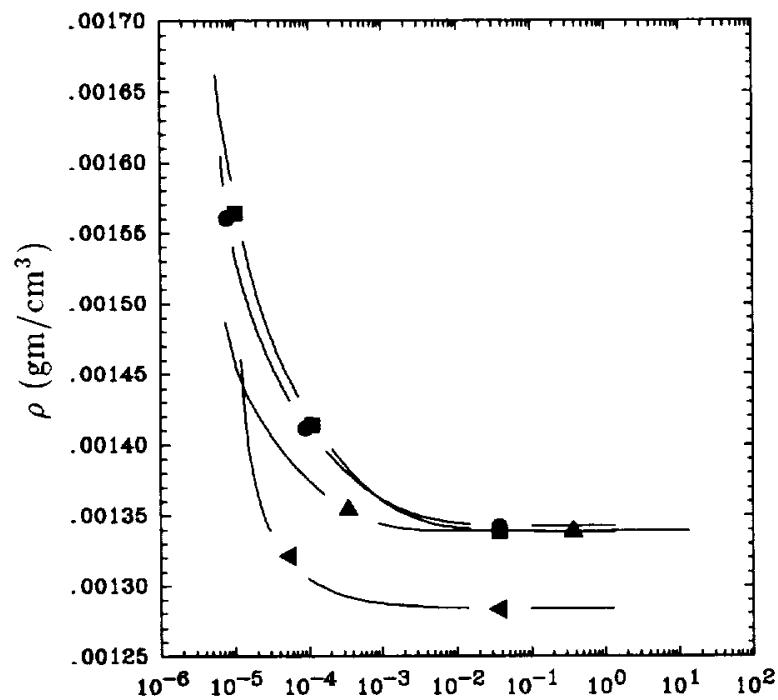

$\Delta t$

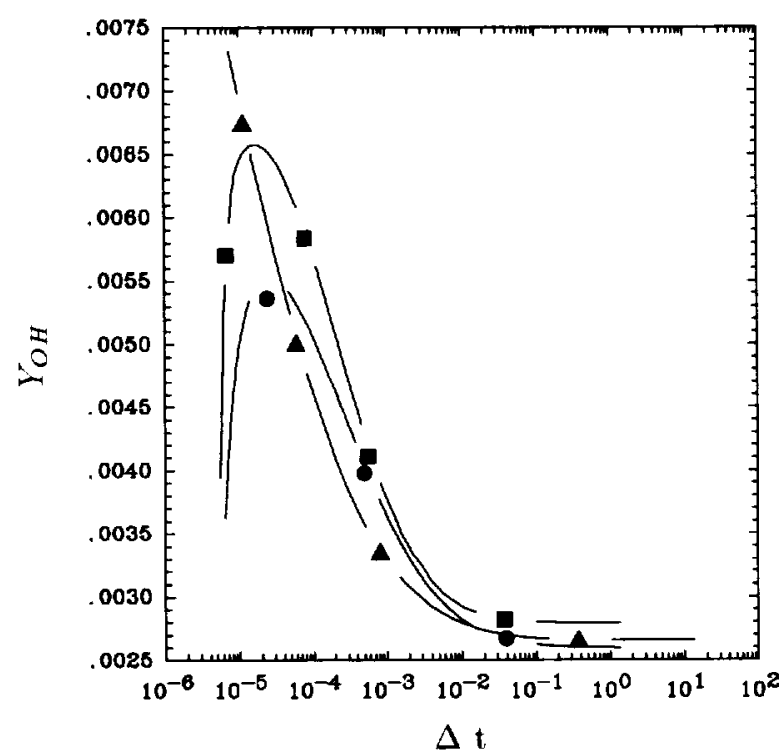

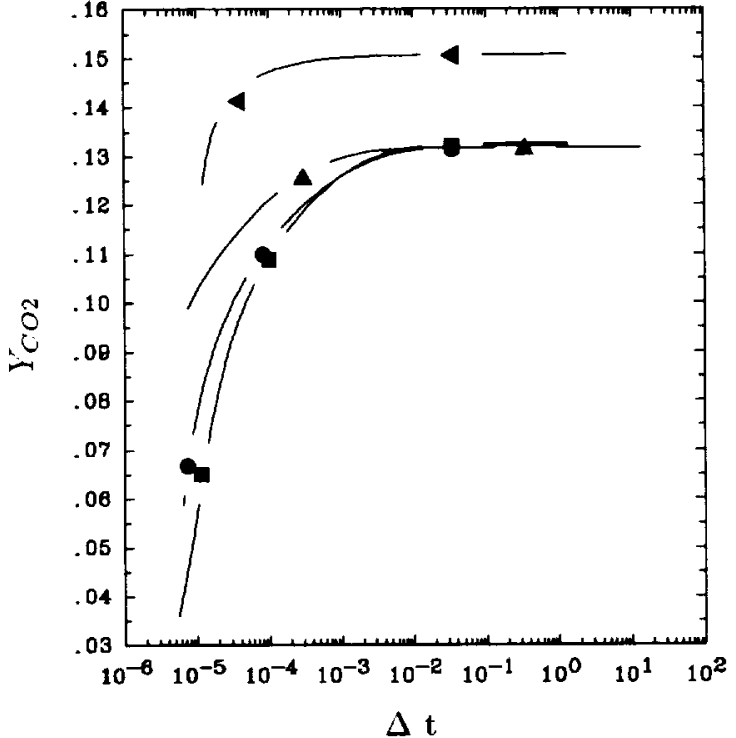

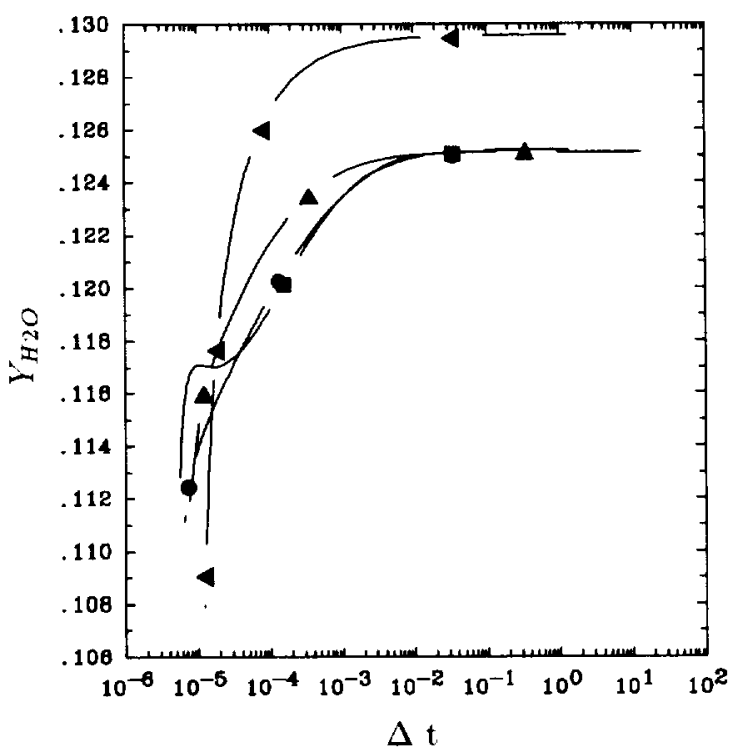

Figure 3: Comparison of different mechanisms for stoichiometric methane-air mixture. PSR results shown as composition quantites plotted against residence time. $O$ - Full mechanism; $\square$ - ILDM table; $\triangle$ 12-species, 10-step mechanism; $\triangleleft-0.01$ Westbrook and Dryer 1-step mechanism. 

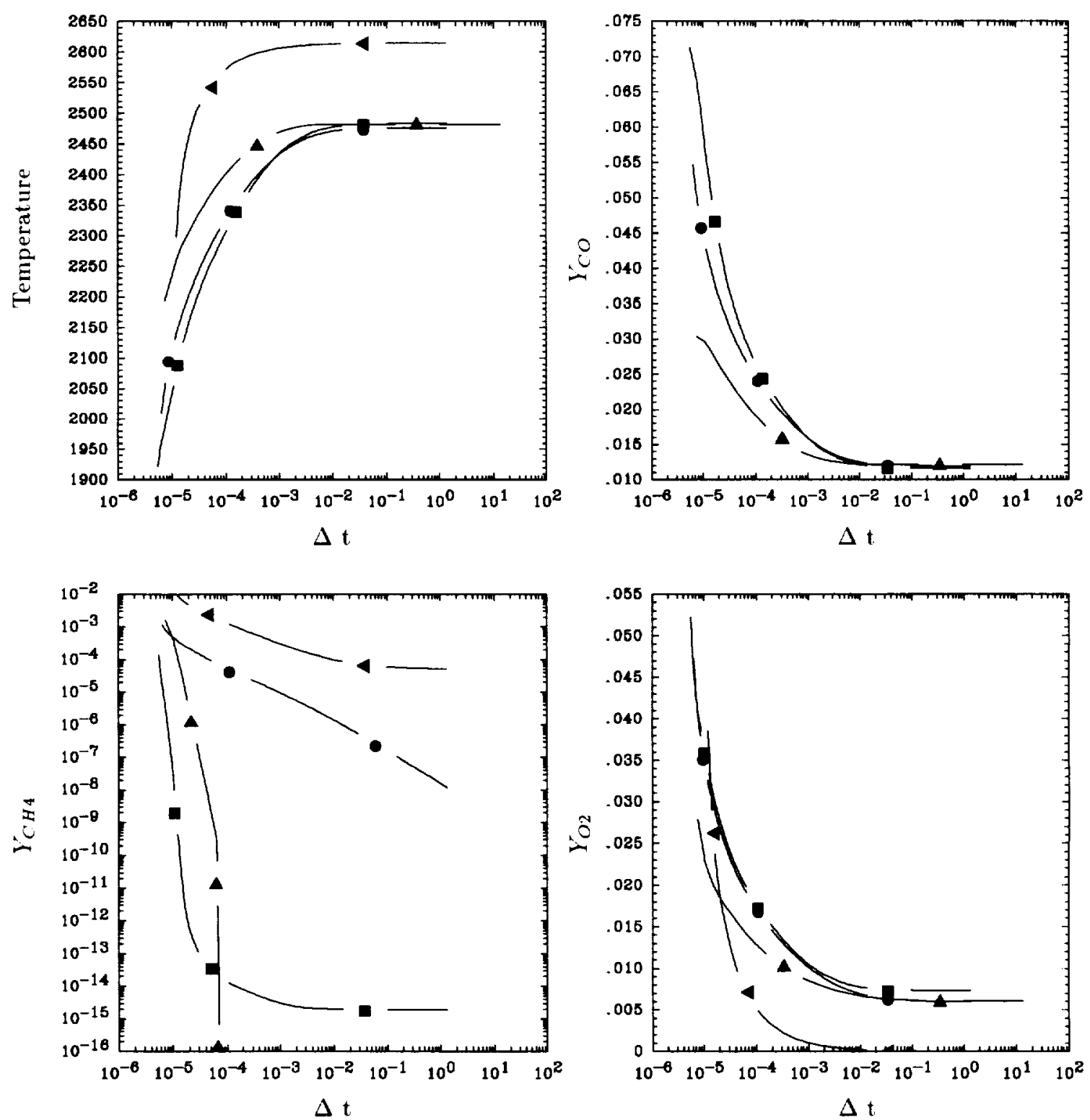

Figure 4: Comparison of different mechanisms for stoichiometric methane-air mixture. PSR results shown as composition quantites plotted against residence time. $\bigcirc$ - Full mechanism; $\square$ - ILDM table; $\triangle$ 12-species, 10-step mechanism; $\triangleleft-0.01$ Westbrook and Dryer 1-step mechanism. 

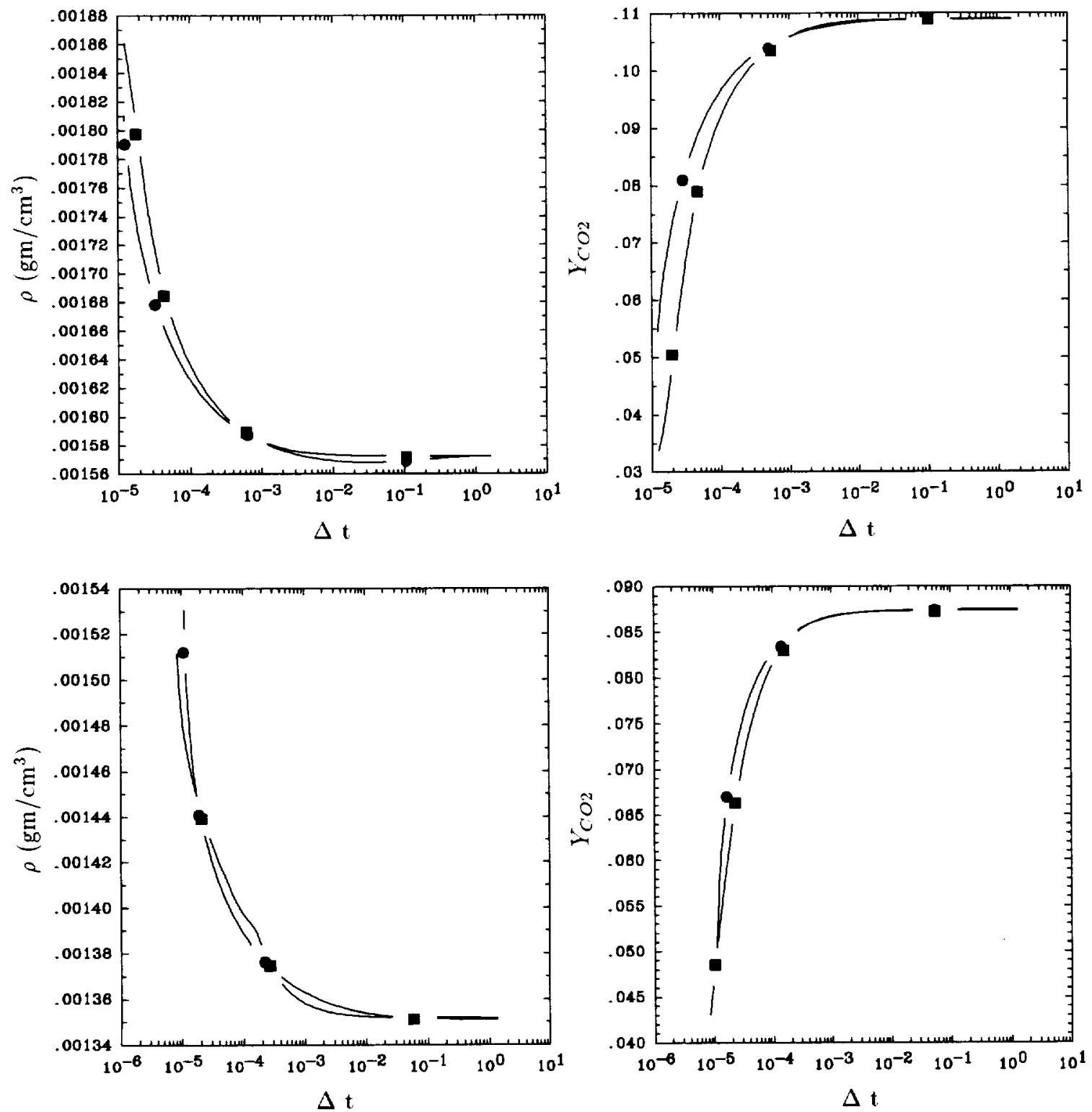

Figure 5: Performance of the ILDM mechanism for lean and rich mixtures of methane-air. PSR results shown as composition quantites plotted against residence time. $\bigcirc$ - Full mechanism; $\square$ - ILDM table. Lean mixture with $\xi=0.04$ shown in upper row. Rich mixture with $\xi=0.07$ in lower row. 

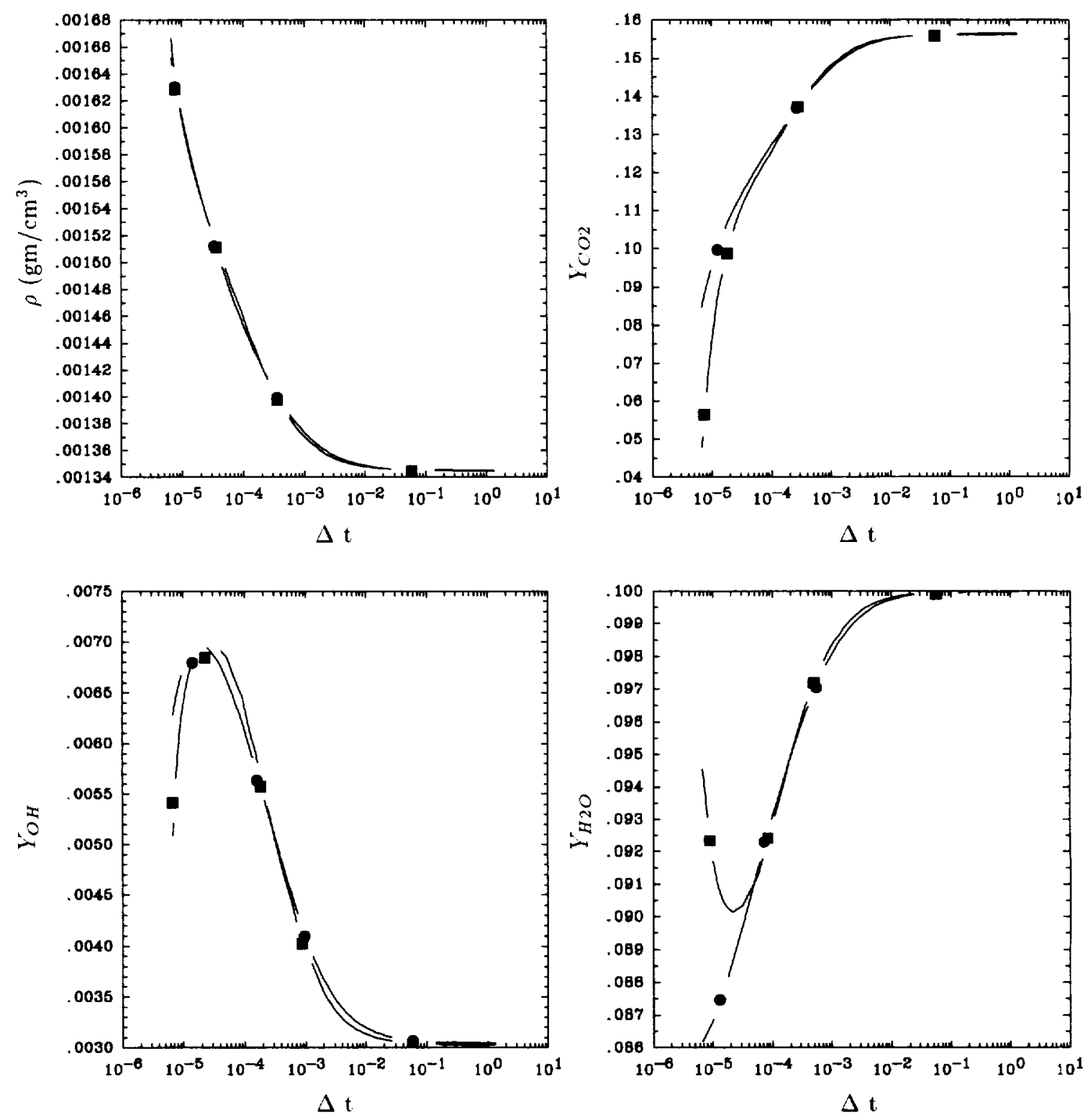

Figure 6: Performance of the ILDM mechanism for stoichiometric mixture of propane-air. PSR results shown as composition quantites plotted against residence time. $\bigcirc$ - Full mechanism; $\square$ - ILDM table. 

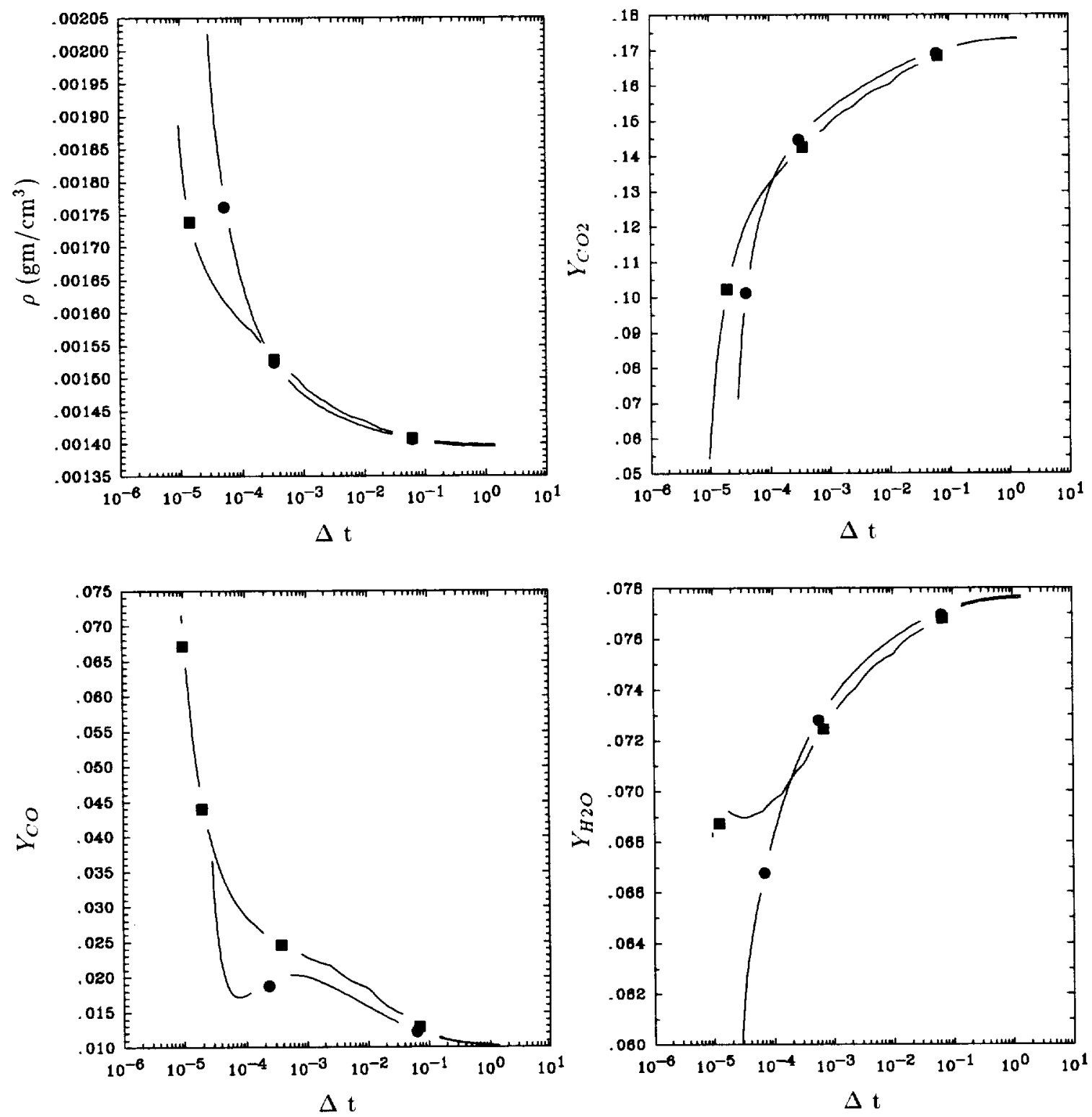

Figure 7: Performance of the ILDM mechanism for stoichiometric mixture of JetA-air. PSR results shown as composition quantites plotted against residence time. $\bigcirc$ - Full mechanism; $\square$ - ILDM table. 


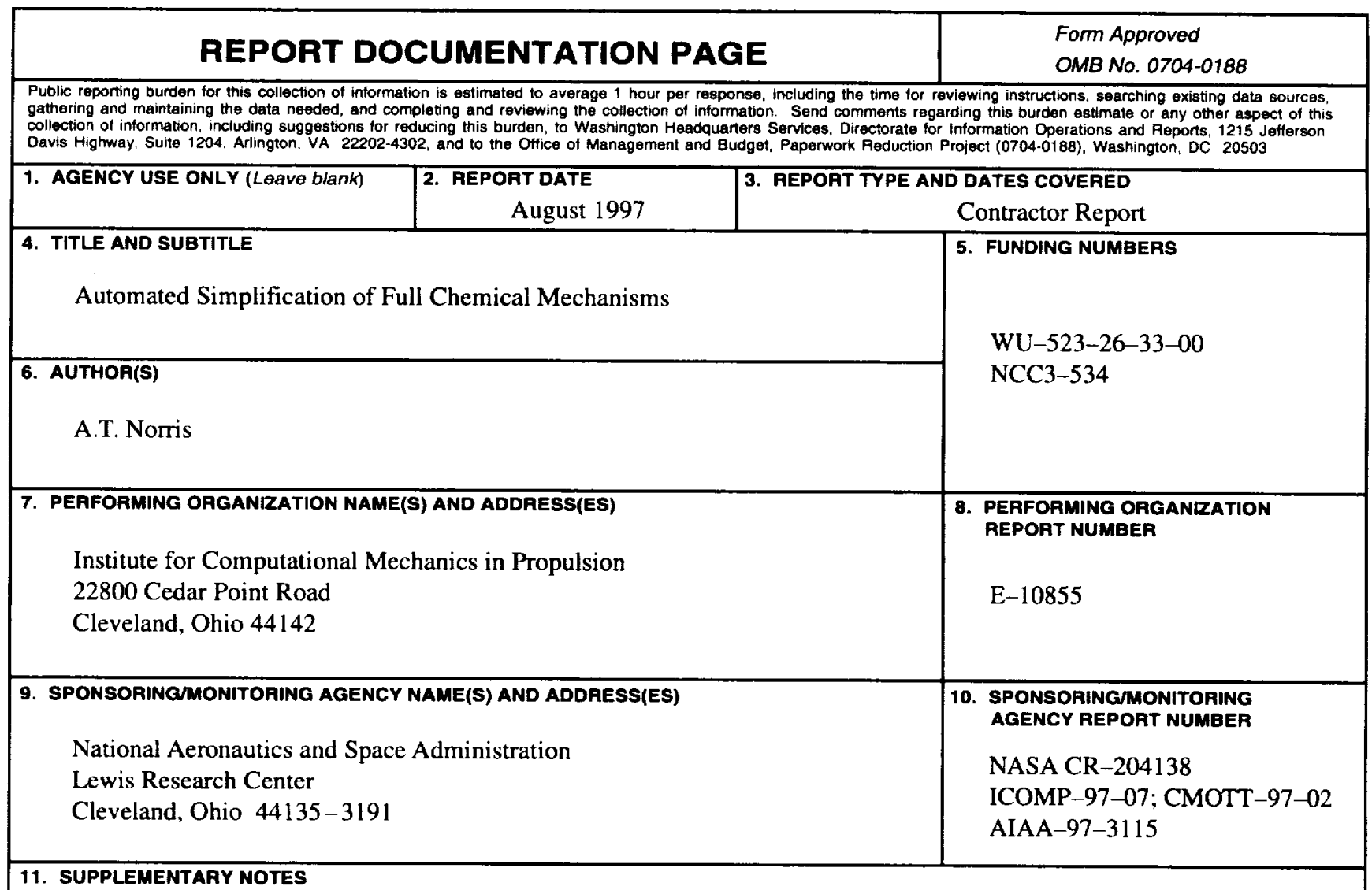

ICOMP Program Director, Louis A. Povinelli, organization code 5800, (216) 433-5818.

\begin{tabular}{|l|l}
\hline 128. DISTRIBUTIONAAVAILABILITY STATEMENT & 12b. DISTRIBUTION CODE
\end{tabular}

Unclassified - Unlimited

Subject Category 34

This publication is available from the NASA Center for AeroSpace Information, (301) 621-0390.

13. ABSTRACT (Maximum 200 words)

A code has been developed to automatically simplify full chemical mechanisms. The method employed is based on the Intrinsic Low Dimensional Manifold (ILDM) method of Maas and Pope. The ILDM method is a dynamical systems approach to the simplification of large chemical kinetic mechanisms. By identifying low-dimensional attracting manifolds, the method allows complex full mechanisms to be parameterized by just a few variables: In effect, generating reduced chemical mechanisms by an automatic procedure. These resulting mechanisms however, still retain all the species used in the full mechanism. Full and skeletal mechanisms for various fuels are simplified to a two dimensional manifold, and the resulting mechanisms are found to compare well with the full mechanisms, and show significant improvement over global one step mechanisms, such as those by Westbrook and Dryer. In addition, by using an ILDM reaction mechanism in a CFD code, a considerable improvement in turn-around time can be achieved.

14. SUBJECT TERMS

15. NUMBER OF PAGES

Combustion; Kinetics; Reduced mechanisms; Manifold methods

\begin{tabular}{|c|c|}
\hline $\begin{array}{c}\text { 17. SECURITY CLASSIFICATION } \\
\text { OF REPORT }\end{array}$ & $\begin{array}{c}\text { 18. SECURITY CLASSIFICATION } \\
\text { OF THIS PAGE } \\
\text { Unclassified }\end{array}$ \\
Unclassified
\end{tabular}

NSN 7540-01-280-5500
19. SECURITY CLASSIFICATION

OF ABSTRACT

Unclassified

\section{LIMITATION OF ABSTRACT}

